\title{
e-Migrinter
}

$18 \mid 2019$

L'ethnographie en migration(s)

\section{Post-colonialisme, hyper-sécurisation des frontières et ethnographie}

Entretien avec Jacinthe Mazzocchetti

Amandine Desille, Bochra Manaï et Aurore Vermylen

\section{OpenEdition}

\section{Journals}

Édition électronique

URL : https://journals.openedition.org/e-migrinter/1628

DOI : 10.4000/e-migrinter. 1628

ISSN : 1961-9685

\section{Éditeur}

UMR 7301 - Migrinter

\section{Référence électronique}

Amandine Desille, Bochra Manaï et Aurore Vermylen, « Post-colonialisme, hyper-sécurisation des frontières et ethnographie », e-Migrinter [En ligne], 18 | 2019, mis en ligne le 11 septembre 2019, consulté le 20 mai 2021. URL : http://journals.openedition.org/e-migrinter/1628 ; DOI : https://doi.org/ 10.4000/e-migrinter.1628

Ce document a été généré automatiquement le 20 mai 2021

Tous droits réservés 


\title{
Post-colonialisme, hyper- sécurisation des frontières et ethnographie
}

Entretien avec Jacinthe Mazzocchetti

\author{
Amandine Desille, Bochra Manaï et Aurore Vermylen
}

Jacinthe Mazzocchetti est professeure en anthropologie au Laboratoire d'Anthropologie Prospective (LAAP) de l'Université Catholique de Louvain (UCL). Ses travaux ont porté successivement sur la jeunesse burkinabé de Ouagadougou en rêve de mieux vivre ${ }^{1}$, sur les bandes urbaines (les "adolescents en exil") à Bruxelles ${ }^{2}$, et sur les jeunes filles placées par le juge en IPPJ (Institut Public de Protection de la Jeunesse) en Belgique ${ }^{3}$. Plus récemment, ses travaux portent sur les réfugiés, demandeurs d'asile et autres déboutés présents sur l'île de Malte, et plus largement sur l'application des politiques migratoires européennes ${ }^{4}$; ainsi que sur les femmes de la diaspora africaine (plus précisément des femmes issues des anciennes colonies belges). Pour ce dernier projet, elle a contribué à l'édition d'un livre «Plurielles. Femmes de la diaspora africaine " $^{5}$ co-écrit avec ces femmes et où sa place d'anthropologue n'était qu'une voix parmi d'autres. C'est au regard de ces deux dernières expériences que nous l'avons invitée à participer à ce numéro sous la forme d'un entretien.

Dans le contexte européen de montée du populisme, d'hyper-médiatisation et d'hypersécurisation des frontières de l'Europe, et à partir du cas de votre terrain à Malte, comment est-il possible de travailler, d'enquêter et de produire de la littérature scientifique ? Comment travaillez-vous avec la potentialité que vos travaux soient récupérés? En somme, quel est le risque de ce type de terrain?

Ma prise de conscience « qu'aucun texte n'est innocent » est assez ancienne. Dès mes premières publications, $\mathrm{j}$ 'ai été confrontée à des situations difficiles et complexes qui m'ont amenée à être très attentive aux enjeux éthiques particuliers à la discipline anthropologique, les terrains ethnographiques étant situés au plus proche de l'humain, co-impliquant le/la chercheur-e et ses interlocuteur-e-s. Je commencerai donc par quelques digressions sur mon parcours, mais qui sont intimement liées aux choix que je pose aujourd'hui dans mes prises de parole et d'écriture à propos des 
migrations, des politiques en la matière, des récits d'exil en contexte de sécurisation des frontières.

Ma première recherche (en dehors du cadre scolaire) s'intitulait "Ethnocampus $»^{6}$. Sans entrer dans trop de détails, il s'agissait de décrire et d'analyser les conditions d'études et de vie des étudiants et, par la suite, des jeunes diplômés de l'université de Ouagadougou. Financée par la coopération universitaire belge, nous (je réalisais cette recherche au sein d'une équipe et une comparaison de plusieurs pays était attendue) avions cependant toute liberté sur la mise en œuvre et le contenu. Seule obligation, remettre à nos financeurs une copie du rapport final. Quelques mois après ce dépôt, nous apprenons que la coopération souhaite suspendre le soutien octroyé à l'université de Ouagadougou. De la lecture de mon texte, des conditions difficiles, mais aussi des ruses des étudiants comme des enseignants décrites, ils en avaient déduit que l'institution dysfonctionnait au point de ne plus souhaiter la soutenir. Il nous a fallu user de beaucoup de talent de persuasion pour leur faire comprendre que là n'était pas la solution, que les choses ne fonctionnaient pas selon leurs critères, mais que, au vu du contexte, elles fonctionnaient néanmoins... Ils ont fini par changer d'optique. Mais toute jeune, très naïve, cette expérience a été pour moi un premier bouleversement, une prise de conscience que même « un bête rapport que personne ne lira ", ce qui était mon imaginaire à l'époque, pouvait avoir une incidence.

Le deuxième bouleversement conséquent a eu lieu lors de l'édition de mon premier ouvrage "Adolescence rupture » écrit après une enquête de terrain depuis l'intérieur d'un IPPJ (Institut de protection de la Jeunesse) ${ }^{7}$. Pour des raisons diverses, sensibilité du sujet (délinquance des mineurs et société sécuritaire), mais aussi première ethnographie publique d'une institution pour mineurs dits délinquants en Belgique, ministre de la Protection de la jeunesse nouvellement en place..., l'écho dans la presse a été assez conséquent. Je ne l'avais pas imaginé, ni même espéré, et, je n'y étais donc pas préparée. Parmi les nombreuses péripéties par lesquelles je suis passée, je mettrai ici en exergue l'effet provoqué par la médiatisation sur les équipes de travailleurs sociaux avec qui j'avais collaboré. De potentielle alliée (nous avions passé beaucoup de temps ensemble, j'étais parfois devenue confidente, je mettais à jour la complexité de leur métier et les impasses d'un système, mes plus proches interlocutrices, mais aussi l'équipe de direction, avaient relu le manuscrit avant publication sans remarques majeures, etc.), j'incarnais soudain la figure de l'ennemi, celle par l'intermédiaire de qui le projecteur est mis sur les souffrances des jeunes, les débrouilles des travailleurs, les failles d'un système. Si derrière des portes closes, nous pouvions partager certaines interprétations; à la lumière, ils/elles se retrouvaient pointé-e-s du doigt comme participants à un système violent ou, en fonction du type de presse et d'orientation politique, a contrario, comme trop laxistes face à ces jeunes pensés comme déviants 8 .

Enfin, je voudrais également mettre en avant un troisième bouleversement, d'un ordre différent encore. Lorsque je travaillais sur le politique au Burkina Faso, notamment lorsque nous menions notre réflexion sur les questions d'oppositions et de semi-autoritarisme avec Mathieu Hilgers ${ }^{9}$, plusieurs de nos interlocuteurs, pour certains de nos amis, ont été menacés. Trois d'entre eux ont même été agressés physiquement suite aux confidences et aux analyses politiques qu'ils avaient partagées avec nous. Dans ce régime liberticide, nous parler, même dans l'anonymat, même avant ou dehors d'une publication quelconque, était déjà de trop... 
J'ai été confrontée à d'autres expériences par la suite, mais ces trois situations, datées de mes premières années de terrain, m'ont en quelque sorte construite. Elles ont fait de moi l'anthropologue que je suis, très attentive aux enjeux éthiques, médiatiques et politiques des travaux de recherche. Bien que nous soyons bien souvent par nos travaux impuissants à infléchir quoi ce soit de l'ordre du monde, j'ai intimement compris que nos attitudes, ce que nous représentons, peuvent avoir une influence sur la vie de nos interlocuteurs, et, que nos textes, même semi-publiques, nous échappent. Faute de pouvoir contrôler leur destinée, ce qui serait par ailleurs dommage et empêcherait les débats, les discussions, les confrontations qui font grandir et transforment les connaissances et les représentations, il nous faut être au fait de ce que le «faire trace», l'acte de consigner, figer, interpréter, et rendre publique implique.

Pour en revenir à Malte, les personnes avec lesquelles je travaille actuellement, migrants principalement ouest-africains, arrivés par naufrage et non régularisés, sont sur le plan institutionnel et des droits humains extrêmement fragilisés, sans statut légal ou sur le coup de statuts fragiles et limités, tout en étant dans des pratiques de débrouilles, de survie parfois, de vie souvent extrêmement riches. La précarité de leur situation, les risques (enfermement, renvoi forcé...) qu'ils encourent appellent à la prudence. Leur quotidien dans les îles maltaises, pays frontière de l'Europe, est fait de contrastes saisissants entre expériences d'enfermements quasi systématiques et très violentes, exploitations cyniques et abusives de leur force de travail et soirées solidaires et festives qui me plongent, en souvenir, dans les nuits chaudes ouagalaises, entre bissap, bière, poulet et riz sauce.

Bien que les questions migratoires fassent régulièrement la Une, mes travaux sont situés dans une temporalité longue qui, par ailleurs, n'a pas pour focus principal les drames en méditerranée, ou encore les agissements de Frontex, mais celui des imaginaires, des stratégies et des enjeux de reconnaissance. Ceci dit, le bruit médiatique et politique qui entoure mes terrains en rend parfois l'analyse difficilement sereine, tout en étant impossible à évincer tant il pèse sur la vie de mes interlocuteurs. Enquêtant à Malte depuis 2012, j'ai vécu en octobre 2014 un moment révélateur lorsque je pris conscience que j'étais en train de mener mes travaux ethnographiques à l'exacte période de l'opération Mos Maiorum ${ }^{10}$. Cette opération policière et militaire de renseignements s'inscrit dans la gestion sécuritaire des migrations adoptée par l'Union européenne (UE). Elle repose sur des logiques de rafles avec pour effet, outre l'extraction d'informations, l'enfermement et/ou le renvoi d'une partie des migrants interceptés et l'arrestation des nommés «facilitateurs ». Le contexte d'hypermédiatisation et d'hyper-sécurisation des frontières de l'Europe ainsi que de concomitance/concurrence des enquêtes policières, d'une part, ethnographique, d'autre part, m'a amené à affiner mes positionnements éthiques et épistémologiques, notamment à propos des silences en enquête et post-enquête et de la censure, ainsi que des résistances et de l'engagement.

Dans ce contexte de rafle, d'abus et d'extorsion de renseignements, que faire des histoires qui se déposent? Que transmettre des informations sensibles collectées, notamment sur les trajectoires migratoires et les dits passeurs, alors que les enquêteurs de Mos Maiorum étaient en quête de données similaires? Ces témoignages reçus engagent. Il n'est pas possible ici de se dédouaner, lorsque ne rien faire serait 
également une forme d'agir, celle du choix de se taire. Dans ce contexte violent de ses asymétries et des exploitations qui le maintiennent, l'acte de décrire est en soi un acte politique en faveur ou en défaveur de ceux qui croisent notre route. Comment raconter alors la survie qui, dans le contexte maltais et plus largement au sein de l'UE, pour les migrants dits illégaux ne peut être que débrouilles sans trahir ni stigmatiser davantage? Comment relater les bricolages face à l'adversité sans entraver les marges de manœuvre négociées? Dans la situation décrite ci-avant, raconter les ruses empruntées par les migrants privés de voies légalisées d'entrée en Europe au moment même où la campagne Mos Maiorum était à l'œuvre m'est apparu éthiquement irresponsable. Cette prise de position est, bien entendu, reliée au potentiel usage de nos écrits et des risques que comporte leur réception en différentes sphères.

Lorsque que dire les ruses risque de mettre des personnes en danger ou à minima de leur fermer certaines portes, mais aussi de renforcer les opinions et les stéréotypes préétablis, tandis que les taire occulte les pouvoirs d'agir, mais aussi les violences d'État et les violences dans l'entre-soi qui leur répondent : il me semble que parler des ruses sans les décrire, - paradoxe ethnographique -, est un entre-deux ici acceptable, bien que les stéréotypes sur les tricheurs et les criminels que sont soidisant les migrants en rendent malgré tout l'expression délicate et sensible. En effet, d'une part, dans le discours des politiciens et des responsables d'institutions, les ruses ne sont pas interprétées en termes de résistance à un contexte structurellement violent, ni en termes de capacités d'agir ou de marges de manœuvre des faibles, de «tactiques » dans un système stratégique gouverné par des rapports de domination extrêmement puissants ${ }^{11}$, mais en termes criminels. D'autre part, les données sur les trajectoires consignées dans mes cahiers sont aussi celles que recherchent les agents d'État mandatés dans les opérations du type Mos Maiorum, afin de mieux les contrôler et de les entraver. Je ne plaide donc pas pour le silence, au contraire, il y a nécessité et urgence de produire des contre-discours, empiriquement rigoureux, qui passent les murs des médias et des politiques, mais plutôt de la nécessité de trouver à dire sans nuire face à des publics extrêmement précarisés et stigmatisés, expérimentant au quotidien la nudité de la vie et l'incertitude de leur destin. Mes expériences de terrain m'ont ainsi progressivement amenée à comprendre l'importance de décrire et d'analyser la manière dont les politiques publiques sont élaborées, comment elles sont mises en œuvre et la diversité de leurs effets $^{12}$.

Définissez-vous votre rôle de chercheur comme «témoin» des réalités les plus complexes ? La légitimité que vous construisez sur le terrain de Malte ne rend-elle pas votre regard privilégié sur la situation des frontières européennes?

Témoin certainement. Notre présence nous implique. Il me semble difficile d'assister à des violences extrêmes, d'une part, et d'observer les créativités et les résistances, d'autre part, sans en faire, d'une manière ou d'une autre, relais. Notre accès progressif et sensible aux faces cachées du monde et aux intimités plurielles nous engage. Regarder, voir, écouter, entendre, sentir, ressentir sans transmettre n'a pour moi aucun sens. La question devient alors celle du comment, pour qui, avec quels objectifs et, enjeu délicat, de quelle place. Quelle position adopter, comment tenter d'être le plus juste possible, écartelée parfois entre un soi situé, un terrain rigoureux, des enjeux éthiques complexes ? De poser une parole sans pour autant devenir 
«sachant ", celui/celle qui parle à la place de. Parler donc en notre nom propre, nourri-e de nos terrains, de nos lectures, de nos expériences.

Le principe même de l'ethnographie, par le temps long passé sur le terrain, par l'articulation progressive des singularités observées et des contextes sociaux dans lesquels elles sont imbriquées, ce que j'appelle souvent la petite histoire dans la grande et inversement, par la mise à jour des processus sociaux, nous permet une prise de parole à la fois ancrée dans un terrain et une diversité de points de vue, située donc dans un espace-temps défini, mais, simultanément, travaillée par les transversalités d'un lieu et d'une époque, ici en l'occurrence celles des politiques de migrations et d'asile, et, de sécurisation des frontières. Regard privilégié donc, nourri d'expériences co-vécues, de récits, d'observations, de lectures, de débats et de discussions qui permet de parler en connaissance de cause, non pas à la place de, mais aux côtés depuis une position spécifique, à la fois située et rigoureuse.

Certains terrains peuvent admettre que le chercheur considéré comme "outsider" prenne la parole ou porte la parole, tandis que d'autres imposent un recul et une distance. Comment négocier la place du chercheur entre porte-parole et porteur d'un "regard sur" ? La pente qui entraîne l'anthropologue vers une anthropologie militante est-elle inexorable? Entre l'approche au terrain, la relation aux gens et l'écriture: comment se définit alors cette militance?

Quant à la place lors de l'enquête, dans les résultats produits et enfin, défendus, il y a en effet des différences importantes entre les terrains, mais aussi selon les types de collaboration. La chercheure que je suis a parfois été mandatée pour prendre la parole "au nom de», position extrêmement sensible et délicate, quand cette dernière n'était pas possible de façon directe ou inaudible, car étouffée par des rapports de pouvoir violents et/ou profondément vulnérabilisée. Dans ce cas, grâce aux procédés d'anonymisation, j'ai toujours tenté de parler entre humilité et rigueur, depuis les récits et les expériences partagées. D'autres situations, recherches, collaborations m'ont amenée à me tenir davantage à côté, à m'effacer parfois (j'y reviendrai). Enfin, croiser les terrains sur une longue durée me permet également de parler en mon nom propre, depuis cette richesse d'expériences, non limitée à un terrain, un groupe, une situation spécifique.

De manière générale, je ne définirais pas ma posture de militante, mais de doublement impliquée: dans sa méthode (posture défendue par le laboratoire d'anthropologie prospective, voire notamment les travaux de Pierre-Joseph Laurent ${ }^{13}$ ) et dans les enjeux de société, éclairés par les recherches que je mène. Cependant, il est important pour moi, dans ma posture de recherche et dans mes écrits, d'allier rigueur et liberté d'analyse, assortis d'une éthique de responsabilité, et non pas de défendre une cause à tout prix. Ce sont les situations de terrains qui m'amènent à prendre position en résonance avec ce que je suis, certainement, mais aussi parfois en dissonance, aidée en cela par le travail de déconstruction et de basculement ${ }^{14}$, de réflexivité et de connaissance de soi opéré. J'ai souvent abordé mes terrains avec beaucoup de naïveté politique en quelque sorte. Quand j'ai démarré l'enquête en IPPJ, l'institution qui m'a ouvert ses portes était considérée dans le secteur comme « la Rolls Royce des institutions ». Je n'y connaissais pas grand-chose et mon regard a d'abord été orienté par cette assertion. C'est peut-être aussi le contraste entre les réalités de terrain et de discours qui m'ont amenée à « ouvrir les yeux », comme on le dit au Burkina Faso. Pays que j'ai également abordé nourrie des discours idéalistes qui circulaient dans le monde estudiantin et celui de la 
coopération au développement : pays des hommes intègres, vitrine de la coopération au développement... Dans une autre mesure, peut-être un peu moins naïve avec l'âge, quand j'ai commencé à travailler auprès des diasporas africaines en Belgique, je ne mesurais pas le degré de racisme structurel dans notre société. Mes questions de départ se transforment souvent radicalement, les terrains me transforment et m'amènent à m'engager dans le débat public de façon assez tranchée, mais en tentant de ne pas gommer la complexité, de ne pas idéaliser ou trop simplifier les faits observés.

Lorsque je dénonce par exemple la militarisation des frontières et ses effets sur les migrants, mais aussi sur celles et ceux qui se pensent du bon côté des barrières et des droits, que l'on dit « autochtones » et plus légitimes à habiter le monde de par ce fait, c'est une réflexion sur les déséquilibres globaux que je mets à jour et face auxquels je me positionne. D'un point de vue plus micro, ethnographique, cela ne doit pas (autant que faire se peut) nous amener à oblitérer les inventivités quotidiennes et les rapports de force à l'intérieur des différents groupes étudiés. Il ne s'agit pas de penser en termes de gentils et de méchants, mais de saisir les processus qui travaillent les uns et les autres. Qu'est-ce qui peut amener un responsable politique à me dire "On ne peut tout de même pas les remettre à la mer »? Qu'est-ce qui peut amener le refus d'un sauvetage ? Comment se construisent ces discours, ces prises de positions, quelles pratiques en résultent? Pourquoi envoyer un adolescent seul dans un périple mortifère? Pourquoi payer des sommes folles pour embarquer sur un canoé pneumatique? Pourquoi violer les femmes dans un campement? Comment les collectifs s'organisent-ils, quelles tensions, quels leaders, quels discours, etc.?

Toute prise de parole est de façon inhérente située, mais il est vrai que, en fonction des thématiques, mais aussi des méthodes, certains terrains nous impliquent plus que d'autres. Davantage encore, il me semble que sur certains sujets, tels que les violences aujourd'hui faites aux migrants, de plus lorsque la méthodologie d'enquête est celle de l'ethnographie, et, donc de la rencontre, un total désinvestissement dans la problématique pourrait à son tour être éthiquement questionnable. L'interprétation dynamique et prospective dans son dialogue permanent entre le local et le global et son attention aux rapports de force, aux asymétries tout autant qu'aux marges de manœuvre, pousse à prendre position, en ce sens qu'elle mène à décrire les paradoxes, les violences, les ambivalences et les hypocrisies, les tactiques, tout autant que les stratégies des dominants. De fait, le chercheur peut-il taire au niveau global, comme au niveau local, un contexte de profondes inégalités, un contexte d'hégémonies politiques, économiques et culturelles cumulées qui se répercutent sur les personnes considérées comme illégales ou en attentes d'une réponse quant à leur statut juridique? Il ne s'agit donc pas de se positionner d'emblée du côté des populations minorisées, en omettant la complexité des rapports sociaux et des relations individus-État, la complexité des rapports de force et leurs logiques dialogiques ainsi que les marges de manœuvre et les résistances négociées, mais de ne pas s'aveugler néanmoins sur les déséquilibres et les rapports de pouvoir à l'œuvre.

Les moments ethnographiques et les histoires de vie partagés nourrissent une certaine urgence à décrire les effets dévastateurs des politiques actuelles à l'encontre des migrants. Une certaine urgence à réinscrire les processus observés en contexte d'exploitation desdites ressources naturelles et des humains, aujourd'hui nommés 
ressources également, dans une logique de profit bien souvent aveugle aux inévitables dommages collatéraux, contexte également de crise multifactorielle et de mise en tension des précaires les uns vis-à-vis des autres. Les mises en route et en danger ne peuvent être saisies en dehors des violences multiples desquelles l'Occident participe indéniablement. Parmi la littérature abondante relative aux questions d'implication ou d'engagement, je reprendrai la distinction proposée par Abélès dans son ouvrage "Penser au-delà de l'État ${ }^{15}$ ». Dans cet ouvrage, il met en tension l'anthropologue militant a priori, rivé à une cause, qui est donc porte-parole, et, l'anthropologie publique où l'anthropologue serait, sans perdre rigueur et liberté d'analyse, un témoin privilégié engagé. Engagement qui n'est pas donc pas le fruit d'un parti pris à priori, mais résulte en quelque sorte d'une prise de parti a postériori, au vu des éléments mis à jour via l'enquête ethnographique. Parti pris a postériori qui naît de l'expérience ethnographique. Il ne s'agit pas de tomber dans le piège de parler à la place de, d'occulter non plus la complexité, mais de prendre néanmoins une parole en propre suite à ce qui a été vécu sur le terrain et d'aiguiser sa capacité critique ainsi que de rendre lisible les violences et de les interroger.

Dans un contexte où les théories postcoloniales ont permis de mieux cerner certaines ambiguïtés face aux terrains de recherche, comment envisagez-vous par exemple la "blanchité" du chercheur? Rend-elle impossible de faire certains terrains ? Implique-t-elle une légitimité de certains chercheurs par rapport à d'autres? N'y a-t-il pas au final autour de cette problématique également des concurrences dans l'accès à la production du savoir et aux institutions universitaires?

Je ne parlerais pas d'impossibilité en tant que telle, mais dans certains cas en effet, la "blanchité », même consciente et analysée, est une donnée qui peut faire entrave. Ce qui est intéressant à étudier et à comprendre en soi. La question de la légitimité est centrale et elle est aussi politique. Face à des populations minorisées, il est essentiel de saisir la manière dont nous sommes situés dans les rapports de force existants, la sensibilité des débats, les rancœurs accumulées. La violence symbolique inhérente à la position de chercheur-e blanc-he implique une réflexivité consciente et exigeante. Il me semble également que nous sommes invités à repenser la pluralité intrinsèque de chaque être humain, à reconnaître les espaces de partage et de rencontre, les lieux du commun et ceux de la divergence. Si nul ne peut être réduit à son corps et si l'expérience ne se suffit pas en soi, nos positions sociales et nos vécus singuliers sont néanmoins inscrits dans nos corps et nos psychés. Travailler ensemble, s'écouter, se lire, débattre, c'est aussi sentir, percevoir là où on se retrouve, là où on ne peut qu'écouter nos vécus sensoriels différenciés et nos expériences singulières inscrites dans des rapports sociaux et des rapports de force qui nous préexistent, mais que nous participons aussi à façonner.

Ceci dit, les milieux proches des théories postcoloniales, subalternes et, de plus en plus, décoloniaux sont aussi très divers. Pour certains groupes d'activistes, de chercheur-e-s, d'artistes..., les «Blancs» ne peuvent pas comprendre ce qu'ils/elles vivent, car incapables de ressentir dans leur corps, leur psyché les rapports hégémoniques passés et actuels, mais aussi parce que confrontés différemment au quotidien aux injustices et aux insécurités, fruits du racisme structurel. Si je partage cette analyse des corps singuliers et sociaux situés qui nous rappelle la dimension subjective de toute prise de parole, mais aussi de toute recherche, je ne pense pas pour autant qu'il ne soit plus possible de travailler ensemble, de chercher à se comprendre, de s'approcher des représentations du monde les uns des autres, ce qui 
reviendrait à remettre en cause la possibilité même de l'ethnographie. Ainsi je n'ai pas plus vécu le suicide sur lequel j'ai travaillé, que l'exil ou la condition noire, mais je m'en suis approchée par des rencontres, un travail sur moi intense, des bouleversements et des basculements progressifs.

Le piège serait de penser, par exemple, qu'il suffit d'être femme pour analyser le patriarcat. La logique scientifique est autre et celle de l'ethnographie suppose de confronter sa propre histoire à celles d'autres, de confronter des histoires et des situations les unes aux autres et d'en relever des transversalités fortes et des tensions, de mettre à jour des processus. C'est comme si je me disais spécialiste des migrations italiennes, parce que mon père a cette histoire, ou polonaises, en relation à ma mère. Leurs récits et leurs vécus ne sont qu'un tout petit bout d'une histoire sociale que je maîtrise peu et que je ne connais de manière sensible qu'à travers eux. Il en résulte un regard très centré, dès lors que l'ethnographie invite à se décentrer, à s'étonner, à se confronter à d'autres, quels qu'ils soient. Les décalages ne sont par ailleurs pas toujours ceux que l'on croit. J'ai par exemple ressenti un sentiment d'étrangeté plus grand face à des adolescentes ayant attenté à leur vie de manière parfois très violente et récurrente que face à des étudiants burkinabè en lutte pour obtenir davantage de droits, de reconnaissance et de liberté. Ce sentiment d'étrangeté m'a aussi souvent davantage envahi passé les murs d'une prison que ceux d'un maquis ouagalais. Le trouble et la difficulté à poser mon corps de manière juste est pour moi bien plus complexe dans un espace confiné, surveillé, gris, que dans un espace ouvert, où je retrouve des amis, burkinabè, mais aussi sociologues, philosophes, artistes qui partagent ma rage politique et mon bonheur de la danse....

Cependant, il y a dans les débats contemporains sur les légitimités à dire quelque chose de fondamental à comprendre. Les populations minorisées, dont les voies ont été largement confisquées, luttent aujourd'hui pour se faire entendre. Elles n'ont pas besoin de notre aide, mais bien d'un soutien solidaire et égalitariste. Elles n'ont pas besoin que nous parlions en leur nom, que nous occupions une fois encore le devant de la scène. Pour moi, il s'agit d'apprendre des autres, de sentir en fonction des enjeux académiques, politiques, militants, la place à occuper. Et parfois, je pense qu'il faut savoir s'effacer. Pour donner un exemple, suite à la recherche et à l'édition de l'ouvrage PluriElles ${ }^{16}$, cette mise en avant de récits de femmes des diasporas africaines en Belgique, nous avons notamment été reçues au parlement wallon. Via ce projet, j'ai eu la chance d'être associée à une cause que je trouve juste, qui me porte, mais lorsqu'il s'agit de défendre leurs droits, les femmes avec qui j'ai travaillé n'ont pas besoin de moi. Je suis, en ce cas, de l'arrière-scène. J'écoute et j'apprends des débats et des discussions qu'elles initient.

Il est vrai également que, comme dans tous milieux, des luttes de pouvoir prennent place au sein des diasporas. Les logiques de reconnaissances individuelles et collectives s'entrecroisent. Les perceptions des luttes et des moyens (pour arriver souvent à des fins similaires) ne sont pas toujours identiques. Certain-e-s, face à la surdité des suprématies économiques, politiques et académiques blanches, pensent que l'unique salut est celui des entre-soi (pensés souvent comme une étape nécessaire et rarement comme une finalité), avec très peu de tolérance pour le discours contradictoire. D'autres sont beaucoup plus nuancés. En fonction des générations, des socialisations, des modèles de lutte, des différences importantes s'énoncent. Probablement y a-t-il aussi parfois des enjeux de concurrence dans l'accès à la 
production du savoir et aux institutions universitaires, mais ces scènes sont actuellement tellement déséquilibrées, en termes de présence physique et intellectuelle (d'autres traditions de pensées), les rapports de pouvoir établis jouant contre les personnes racisées, que ces luttes en sont pour moi non seulement légitimes, mais nécessaires.

La positionnalité différente influence sur la production de connaissances, c'est une évidence. On a donc besoin de croiser les recherches et les connaissances, d'apprendre les uns des autres. Plus il y a de diversité, plus on s'approche de la complexité des rapports humains. Pour le moment, les lieux universitaires sont encore largement hégémoniques dans leurs rapports aux autres, non occidentaux, et aux autres savoirs, de traditions multiples (postcoloniale, décoloniale, subalterne, féministe...). Saisir ces connaissances à partir de leur lieu spécifique, ouvrir le dialogue sur des bases respectueuses et non dans l'imaginaire de la détention du paradigme de ce que serait la production de connaissances me semble indispensable. Pour être honnête, mes lectures de penseur-e-s, écrivain-e-s, activistes noires ont fondamentalement changé mon approche de toute une série de questions, notamment par leur mise en exergue de la profondeur historique des rapports de domination et des résistances, par le focus davantage sociétal de leurs approches, par les questions spécifiques qu'ils/elles posent, par des entrées souvent non disciplinaires...

Comment "échapper" ou "faire avec" les relations héritées du postcolonialisme ou encore les relations de genre? Pour que le terrain ne devienne pas impossible ou interdit, ne s'agitil pas plutôt de rendre visible une certaine positionnalité ? L'enjeu, n'est-il pas au fond, de faire du terrain "situé" ? Comment incarnez-vous l'analyse située (lors des entretiens, lors de la restitution, de l'écriture, etc.) ?

En effet, interroger et rendre visible une certaine positionnalité est à la base de la pratique ethnographique. Si, en anthropologie, le tournant postmoderne notamment, et, de manière plus large, les courants postcoloniaux et subalternes ainsi que certains courants féministes ou queer ont débattu longuement de l'incidence de la personne $\mathrm{du} / \mathrm{de}$ la chercheur-e (son milieu social, ses origines, son genre, etc.) sur le savoir produit et provoqué des débats épistémologiques importants au sein de la discipline, le piège serait d'imaginer que cette énonciation de soi puisse s'auto-suffire et faire office de garantie de la véracité de la parole. Ces travaux nous enseignent en effet l'exigence et la délicatesse que suppose un réel retour réflexif. Retour réflexif qui ne se résumerait pas à dire " je suis une femme blanche, en couple, mère... », comme une sorte de clef de lecture offerte au récepteur de l'enquête en imaginant que cette honnêteté réduirait les biais; mais qui aurait comme objectif d'analyser finement l'incidence que ces divers éléments, qui se dégagent de notre personne, a eue sur les rencontres, les relations tissées, et, donc sur les données et leur interprétation, mais aussi sur nos choix de terrains et d'écriture. Démarche qui nous enjoint à aller audelà des évidences et de la surface des catégories sociales, et qui se doit par ailleurs de tenir compte du fait que de nombreux éléments nous échappent: le hasard des rencontres, les affectations et les négociations de terrain, les non-dits et les silences les nôtres tout comme ceux de nos interlocuteurs -, mais aussi nos propres failles, nos propres quêtes qui, tels des volcans endormis, surgissent ou resurgissent parfois au détour d'observations, de mots et de récits sans toujours avoir été explicitement conviés. 
Ce travail est permanent, en fonction des terrains, des rencontres, mais aussi de notre parcours de vie. Il importe ainsi d'être au fait des changements du soi. Il est très différent d'être une "femme jeune et seule » ou d'être une "femme mère ", par exemple. Être «blanche » en Afrique, auprès des diasporas africaines ou en prison a des incidences très différentes. Sur des sujets sensibles, des terrains traversés par la question des légitimités et des hégémonies, la tâche (ce retour réflexif) est parfois plus difficile encore. Il est important d'être au clair avec ses motivations de recherche. Pourquoi travailler sur la condition noire alors que d'autres, considérés comme plus légitimes, y excellent ? J'ai insisté déjà sur la richesse de la pluralité des connaissances produites, mais j'ajouterai, en empruntant les mots de Reni EddoLodge que " le racisme est [aussi] un problème de Blancs » ${ }^{17}$.

Dans la pratique ethnographique, les outils principaux qui permettent ce travail réflexif sont le cahier de terrain, mais aussi les groupes d'intervision, et, enfin les discussions analytiques et méthodologiques avec les personnes du terrain: les confrontations de points de vue, l'acceptation des bouleversements induits par les rencontres, les basculements progressifs, petit pas d'une transformation du soi et d'un accès non idéalisé et non hégémonique à l'autre. Dans l'écriture qui fait suite à nos terrains, il est important d'énoncer qui l'on est, d'où l'on parle, comment nos interprétations sont advenues. Il s'agit d'adopter une position à la fois sensible et rigoureuse qui permet de penser à partir de, avec le terrain, depuis les observations et les récits. Il est important de donner à voir notre cheminement intellectuel et ses ressorts empiriques. Sans prendre trop de place, sans devenir le héros de son propre texte, faire de l'autobiographie, ce qui est un autre projet, je pense qu'il est également important d'énoncer nos limites. Cela nous éloigne du post-colonialisme, mais lors de l'enquête en IPPJ, par exemple, je ne pouvais taire dans mon écriture, la sensation d'étouffement permanente ressentie sur le terrain qui, malgré un travail conséquent et minutieux, était indéniablement présente dans le regard posé.

Au final, on en revient toujours à quelques mots clefs identiques : réflexivité, rigueur analytique, épaisseur empirique, implication, humilité et éthique. Ce n'est pas une recette, mais des balises que je suis autant que faire se peut. Par ailleurs, en fonction des contraintes éditoriales, tous ces aspects ne se retrouvent pas toujours énoncés dans un même texte. Les livres permettent ce que les articles cadenassent parfois, pensées morcelées en divers papiers. Pour en revenir aux relations héritées du postcolonialisme, chaque terrain, chaque rencontre, chaque altercation également est l'occasion d'avancer un peu plus sur les implications de la positionnalité blanche sans oublier de la penser de manière intersectionnelle. Les rapports de pouvoir sont aussi genrés, de classe, d'âge également. En fonction des scènes, je suis du côté des minorisés ou des dominants (peu importe ce que j'en pense et ce que j'en fais, l'histoire me place à cet endroit) et cette conscience est un outil de travail précieux.

Au regard de vos expériences passées, comment envisager une continuité de votre relation aux participants à la recherche ethnographique? Que penser de la co-écriture invitant les participants à se mettre eux-mêmes en récit? Avez-vous expérimenté des problèmes lors de cette expérience?

Il y a plusieurs manières de répondre à cette question. Les relations de terrain sont multiples, de l'amitié à l'éviction en passant par la colère. D'un point de vue personnel, de chaque terrain me reste des amitiés fortes dont l'étendue dépasse largement celle des relations de travail. D'un point de vue professionnel, il m'est 
arrivé à plusieurs reprises de penser des pistes d'intervention en collaboration avec des personnes du terrain, mais aussi de porter ensemble sur la scène publique des résultats de recherche.

«Écrire avec» revêt pour moi différentes significations. Avec conscience des rapports de force, des hégémonies et des violences épistémiques. Avec conscience de son propre corps biologique et social. Avec rigueur et humilité. Avec comme sources de réflexion et de connaissance des auteur-e-s subalternes et racisé-e-s. Les savoirs émanant d'expériences sensibles d'altérisation et de vécus de subalternité, tout autant que de luttes, en déplaçant radicalement les points de vue, offrent aux sciences humaines et sociales de nouvelles épistémologies et théories qui permettent de saisir le monde de façon complexe et renouvelée. Avec les personnes, co-auteur-es d'un même texte, comme ce fût le cas dans le projet « PluriElles ", en proposant des démarches d'écriture inclusive et collaborative qui combinent co-responsabilités et liberté de pensées.

Co-écrire est une démarche à la fois riche et difficile. Une recherche peut être collaborative sans impliquer que les plumes soient conjointes. Travailler sous forme de longs récits de vie, de dynamique d'entretiens collectifs sur la durée peut être détaché du texte final produit. Je distinguerais donc les logiques de co-construction (ce qui me semble être toujours le cas dans les recherches ethnographiques, mais à des degrés divers) de celles de co-écriture. La co-écriture implique à minima, lorsque la plume, la mise en texte final est dans les mains $\mathrm{du} / \mathrm{de}$ la chercheur-e, un travail de relecture et de validation du texte produit, et, de responsabilité commune du contenu mis en circulation. J'ai d'ailleurs vécu l'expérience avec des travailleurs sociaux impliqués dans une dynamique collaborative qui, en bout de course, ont refusé de voir leurs noms apparaître, non pas en désaccord avec le texte produit, mais par crainte des implications potentielles, plus forts derrière l'anonymat pour se le réapproprier ensuite et l'utiliser en étant davantage protégés ${ }^{18}$.

Le projet «PluriElles » auquel vous faites référence est différent. Ici les femmes dont nous avons retracé les récits de vie sous forme de portraits photographiques et littéraires ont accepté de rendre une part de leur vie publique. Les textes publiés sont littéralement co-écrits, signés de deux plumes. N'étant pas protégées (leur nom, leur visage), nous avons dû davantage discuter, négocier parfois, ce qui pouvait être dit ou pas, ainsi que la manière d'aborder certains sujets. La trame ici est largement pensée en commun. Nous sommes actrices des textes ensemble. Textes qui ont donc leur part d'ombres et de mystères. Ce qui n'empêche en parallèle, mais sous le couvert de l'anonymat, de décrire et de réfléchir ensuite aux parts secrètes qui se sont déposées lors des entretiens, mais que les femmes n'ont pas souhaité publier: des bribes d'intimité (famille, couple, enfants), mais aussi le racisme vécu dans les corps (les cheveux, la peau, l'odeur) ... Je ne parlerai donc pas de problèmes en tant que tels, mais des contraintes liées à la dynamique de recherche et d'écriture choisie. Il en résulte un ouvrage qui nous co-appartient, au sein duquel se déploient également des divergences. Un ouvrage porté ensemble et dont je peux m'effacer, en fonction des scènes, de sa réception publique.

Je suis actuellement impliquée dans de nouveaux projets qui portent plus loin encore cette dynamique de mise en récit de soi, de ses expériences, de ses pratiques, notamment via l'animation d'ateliers d'écriture. Je ne l'opposerais pas à celle de 
l'observation participante, faire avec, vivre avec et surtout tisser des relations profondes, sentir/ressentir ce qui fait monde pour l'autre, le trouble, le porte passent par ce vivre ensemble; mais amener les personnes à se mettre en écriture (ethnographique, poétique ou fictionnelle - j'ai et je continue à expérimenter les trois) est une autre manière de basculer vers leurs mondes, leurs intimités profondes, leurs imaginaires au-delà des discours, et, donc, de produire et de diffuser des connaissances.

\section{NOTES}

1. Mazzocchetti, Jacinthe (2009) Être étudiant à Ouagadougou. Itinérances, imaginaire et précarité, Paris, Karthala, 360 p. (Hommes et Sociétés).

2. Jamoulle, Pascale; Mazzocchetti Jacinthe (2011) Adolescences en exil, Louvain-la-Neuve, Academia, 358 p. (Anthropologie Prospective).

3. Mazzocchetti, Jacinthe (2005) L'adolescence en rupture: le placement au féminin. Une enquête de terrain, Louvain-la-Neuve, Academia-Bruylant, $170 \mathrm{p}$.

4. Voir notamment : Mazzocchetti, Jacinthe (2017) Dire la violence des frontières dans le rapport de force que constitue la procédure d'asile. Le cas d'Ali, de l'Afghanistan en Belgique, Revue Européenne des Migrations Internationales, vol.33, $\mathrm{n}^{\circ} 2$ \& 3, pp.91-114; Mazzocchetti, Jacinthe (2016) The Ethics of Ethnographic Fieldwork in the Context of War against Migrants, Anthropologie \& développement, $\mathrm{n}^{\circ} 44$, pp. 55-78 et Mazzocchetti, Jacinthe (2014) Le corps comme permis de circuler. Du corps-héros au corps-souffrant dans les trajectoires migratoires et les possibilités de régularisation, Parcours anthropologique, $\mathrm{n}^{\circ}$ 9, pp. 133-154.

5. Mazzocchetti, Jacinthe ; Nyatanyi Biyiha, Marie-Pierre (2016) PluriElles. Femmes de la diaspora africaine, Paris, Karthala 184 p. (avec les photographies de Vercheval V.).

6. Mazzocchetti Jacinthe (2000) Les étudiants de l'université de Ouagadougou, in Laurent, P.-J. ; Petit, P.; Poncelet, M. (dir.) L'université africaine: comparaison de trois campus universitaires, Bruxelle, UCL/ULB/ULG, CIUF-AGCD, pp. 179-272 et Mazzocchetti, Jacinthe (2002) Les diplômés de l'université de Ouagadougou, in Laurent, P.-J.; Petit, P.; Poncelet, M. (dir.) L'université africaine: insertion socioprofessionnelle de jeunes diplômés, Bruxelle, UCL/ULB/ULG, CIUF, pp. 119-228.

7. Mazzocchetti, Jacinthe (2005) op. cit.

8. Pour aller plus loin: Mazzocchetti, Jacinthe (2015) Basculements sensibles, implications et écritures, in Defreyne, E. ; Hagdad Mofrad, G. ; Mesturini, S. ; Vuillemenot, A.-M. (dir.) Intimité et réflexivité, Itinérances d'anthropologues, Louvain-la-Neuve, Academia, pp. 83-114.

9. Hilgers, Mathieu ; Mazzocchetti, Jacinthe (dir.) (2006) Le Burkina Faso : l'alternance impossible, Politique africaine, $\mathrm{n}^{\circ}$ 101, 216 p. et Hilgers, Mathieu ; Mazzocchetti, Jacinthe (dir.) (2010) Révoltes et oppositions dans un régime semi-autoritaire. Le cas du Burkina Faso, Paris, Karthala, 324 p.

10. Pour plus de détails sur cette opération, voir : https://enigmur.hypotheses.org/4165

11. Certeau, Michel de (1990) L'invention du quotidien I : Arts de faire, Paris, Folio, 347 p.

12. Pour aller plus loin : Mazzocchetti Jacinthe (2016) op. cit.

13. Laurent, Pierre-Joseph (2011) Observation participante et engagement en anthropologie, in Hermesse, J.; Singleton, M. ; Vuillemenot, A.-M. (dir.) Implications et explorations ethiques en anthropologie, Louvain-la-Neuve, Academia, pp. 45-70, (Anthropologie prospective). 
14. Basculer signifie ici, au travers d'une expérience de décentrement, de rencontres et de relations, à la fois le bouleversement qui résulte de la transformation de soi inhérente à l'ethnographie et la capacité progressive de comprendre le monde depuis les schèmes de pensées et les référents d'un autre.

15. Abélès, Marc (2014) Penser au-delà de l'État, Paris, Belin, $112 \mathrm{p}$.

16. Mazzocchetti, Jacinthe; Nyatanyi Biyiha, Marie-Pierre (2016) op. cit.

17. Eddo-Lodge, Reni (2018) Le racisme est un problème de Blancs, Paris, Autrement, $296 \mathrm{p}$.

18. Mazzocchetti, Jacinthe (dir.) (2007) Le travail social de proximité en questions. Entre utopie et sombre conscience, Louvain-la-Neuve, Academia-Bruylant, $204 \mathrm{p}$.

INDEX

Mots-clés : réfugiés, demandeurs d'asile, politique migratoire, femme

Index géographique : Malte, Belgique

\section{AUTEURS}

\section{AMANDINE DESILLE}

Chercheuse post-doctorante, IGOT / Universidade de Lisboa

amandine.desille@gmail.com

\section{BOCHRA MANAÏ}

Docteure en Études Urbaines de l'INRS-UCS, enseignante dans des universités montréalaises et est impliquée dans le milieu communautaire à Montréal.

bmanai@uottawa.ca

\section{AURORE VERMYLEN}

PhD student, laboratoire d'anthropologie prospective (LAAP), UCL (Belgique) ; Bourse FRESH

(F.R.S.-FNRS)

aurore.vermylen@uclouvain.be 